\title{
Spy out to Protect: Sensing Devices for Wildlife Virtual Fencing
}

\author{
Rita Anastácio1, Sérgio Cardoso², Mário Jorge Pereira ${ }^{1 *}$ \\ ${ }^{1}$ Departamento de Biologia e CESAM, Universidade de Aveiro, Aveiro, Portugal \\ ${ }^{2}$ AFPR-BB, Aveiro, Portugal \\ Email: *mverde@ua.pt
}

How to cite this paper: Anastácio, R. Cardoso, S. and Pereira, M.J. (2018) Spy out to Protect: Sensing Devices for Wildlife Virtual Fencing. Open Journal of Ecology, 8, 192-208.

https://doi.org/10.4236/oje.2018.83013

Received: February 14, 2018

Accepted: March 26, 2018

Published: March 29, 2018

Copyright (c) 2018 by authors and Scientific Research Publishing Inc. This work is licensed under the Creative Commons Attribution International License (CC BY 4.0).

http://creativecommons.org/licenses/by/4.0/

(c) (i) Open Access

\begin{abstract}
To avoid wildlife-human conflict several solutions are used, like electrical fences, the most expensive solution. Nowadays, technology enables alternative and cheaper approaches for conservation projects. A technological device was developed to detect elephants, moving on their habitat, and predict and react by avoiding confrontation with man. The devices were tested in field experiments, and proved to be efficient in capturing floor vibration, and air-sound signals. Collected data also enabled the estimation of the vibration-source by calculus (using triangulation), revealing the importance of the methodology for real-time location and tracking of high mass animals (e.g. elephants). Building up a mesh of devices, separated $25 \mathrm{~m}$ from each other, is estimated as possible to monitor and identify different animals (by discriminating patterns) in an area, like a virtual fencing system. Though the devices may be effective for animal behaviour research, or even animal communication analysis, or other Biology field, other applications outside Biology are possible for them, like monitoring of: rock-falling, micro seismic railway, infrastructures, and people movements.
\end{abstract}

\section{Keywords}

Micro-Electro-Mechanical Systems (MEMS), Microseismic Detection, Virtual Fencing, Free-Ranging Wildlife

\section{Introduction}

The purpose for using a vibration sensor is related with its specifications and application. There are several types of vibration sensors, and they all have different performances. Geophones, for example, are generally used as ground sensors in seismic studies [1] [2] [3] [4], and can be used to detect elephant 
walking vibrations [5] [6] [7] [8]. Liang and Lin (2013) [9] in their paper about ground vibrations generated by the impact of rocks upon the ground, refer that microphones, seismometers, geophones, accelerometers, hydrophones and fiber-optic sensors are viable technologies to detect ground-vibrations. Ground vibrations are, in fact, mechanical waves, which are defined as a periodic disturbances travelling through a medium [10].

Ground-vibrations can be produced by a walking being, or by an earthquake, or by rocks free falling from a cliff. However, an earthquake releases much more energy than anthropogenic activities on the surface. Seismologists classify seismic events by its magnitude [11] and energy signals from a typical seismic event can be captured by classical geophones, but microseismic events $(M<0-2)$, which result from weak natural tectonic motions or are induced by man-made changes on the surface of the earth [12], can be captured by other type of technologies, not so expensive.

According with a frequency scale, sound waves are categorized into: infrasonic waves (below $20 \mathrm{~Hz}$ ), audible waves $(20 \mathrm{~Hz}-20000 \mathrm{~Hz}$; lie within the range of sensitivity of the human ear), and ultrasonic waves (have frequencies above the audible range) [10]. Also, the properties of a non-vacuum medium (gas, liquid or solid), conditions waves speed, and attenuates waves propagation in different ways [11].

Sound waves, for example, "travel through room-temperature air with a speed of about $343 \mathrm{~m} / \mathrm{s}$, travelling with higher speeds through most solids" [10]. Seismic waves speed depends also on the medium through which they travel, especially its matrix and its porosity. Soil is generally a heterogeneous medium, composed by different layers, with different compositions. Also, the amount of sources that produce microseismic and sound vibrations on the soil surface interferes with ground-wave caption by technological devices [11].

Accelerometers are used as microseismic sensors [4], with potential to capture low-frequency ground signals. Lainé and Mougenot (2014) [13] used micro-electro-mechanical systems (MEMS)-based digital sensors and compared them to traditional coiled geophones, founding advantages and disadvantages in these two technologies. Using its advantages when compared to traditional geophones, and concerned in reducing the logistic complexity and expenditure of the technology to capture low-frequency vibrations produced on the soil surface, this work developed triaxial MEMS accelerometers to detect and capture ground vibrations, especially low-frequency vibrations. It was aimed at testing the feasibility and limitations of this seismic/acoustic monitoring device/system that was named "loxophone".

The new device aims at overcoming issues found in other attempts to do geofencing based on vibration sensing.

\section{Material and Methods}

\subsection{Model for Source Location}

To estimate the location of a source-vibration using stationary stations, we can 
use techniques based on elapsed time or vibration intensity. For example, in the seismic monitor solutions, an elapsed time technique is used to estimate the epicentre of an earthquake. In our case, due to the short distances between the sensors, a vibration intensity solution is a more efficient approach. Advances in electronic sensors, namely, triaxial MEMS accelerometers, gave a significant contribution to implement these solutions at an affordable cost. These modern sensors enable us to sense very small vibrations, with high axial accuracy.

To estimate the location of a vibration source using the intensity received by stationary vibration sensors, we use the mathematical model described below.

Vibration intensity $\left(I_{n}\right)$ at a certain distance $(r)$ is given by the following equation, where $I_{\text {source }}$ is the intensity of the source of the vibration.

$$
I_{n}=\frac{I_{\text {source }}}{\pi r^{2}}
$$

Ground waves propagate in very different ways (P-waves, S-waves, Rayleigh waves, etc.), so $I_{n}$ is given by the vector sum of the intensity sensed on each axis $(x, y, z)$ :

$$
I_{n}=\sqrt{\left(I_{x \_ \text {axis }}\right)^{2}+\left(I_{y \_ \text {axis }}\right)^{2}+\left(I_{z \_ \text {axis }}\right)^{2}}
$$

Considering that the Intensity of the vibration source $\left(I_{\text {source }}\right)$ is the same for all 4 sensors, then:

$$
I_{1} r_{1}=I_{2} r_{2}=I_{3} r_{3}=I_{4} r_{4}
$$

The distance between the vibration source and the sensor $\left(r_{n}\right)$ can be expressed using the coordinates of the location of the source $\left(x_{\text {source }}, y_{\text {source }}\right)$ and the sensor $\left(x_{n}, y_{n}\right)$, as follow:

$$
r_{n}=\sqrt{\left(x_{\text {source }}-x_{n}\right)^{2}+\left(y_{\text {source }}-y_{n}\right)^{2}}
$$

The intensity of the vibration source $\left(I_{\text {souce }}\right)$ is unknown, so we need to estimate the location using the ratio of the intensity received by a pair of sensors (Figure 1):

$$
I_{1} r_{1}=I_{2} r_{2}
$$

Drilling down the Equation (5) using the $x, y$ version of the $r_{n}$, we obtain the Equation (6), where $x_{1}, y_{1}, I_{1}$, are known values from sensor $1, x_{2}, y_{2}, I_{2}$ are known values from sensor 2 .

$$
x_{\text {source }}=\frac{2 x_{1} I_{1}^{2}-2 x_{2} I_{2}^{2} \pm \sqrt{\left(2 x_{2} I_{2}^{2}-2 x_{1} I_{1}^{2}\right)^{2}-4\left(I_{1}^{2}-I_{2}^{2}\right)\left[\left(I_{1}^{2}-I_{2}^{2}\right) y_{\text {source }}^{2}+\left(2 y_{2} I_{2}^{2}-2 y_{1} I_{1}^{2}\right) y_{\text {source }}+I_{1}^{2}\left(x_{1}^{2}+y_{1}^{2}\right)-I_{2}^{2}\left(x_{2}^{2}+y_{2}^{2}\right)\right]}}{2\left(I_{1}^{2}-I_{2}^{2}\right)}
$$

The Equation (6) represents the relation between $x_{\text {source }}$ and $y_{\text {source }}$ variables. This relation can be geometrically represented by a line of all possible points for the location of the source of the vibration. The diagram below (Figure 1) shows this line for a set of $I_{1} / I_{2}$ examples $\left(I_{1} / I_{2}=4.00 ; I_{1} / I_{2}=2.00 ; I_{1} / I_{2}=1.33 ; I_{1} / I_{2}=\right.$ $\left.1.00 ; I_{1} / I_{2}=0.75 ; I_{1} / I_{2}=0.50 ; I_{1} / I_{2}=0.25\right)$, considering the location of sensor ${ }_{1}=$ 


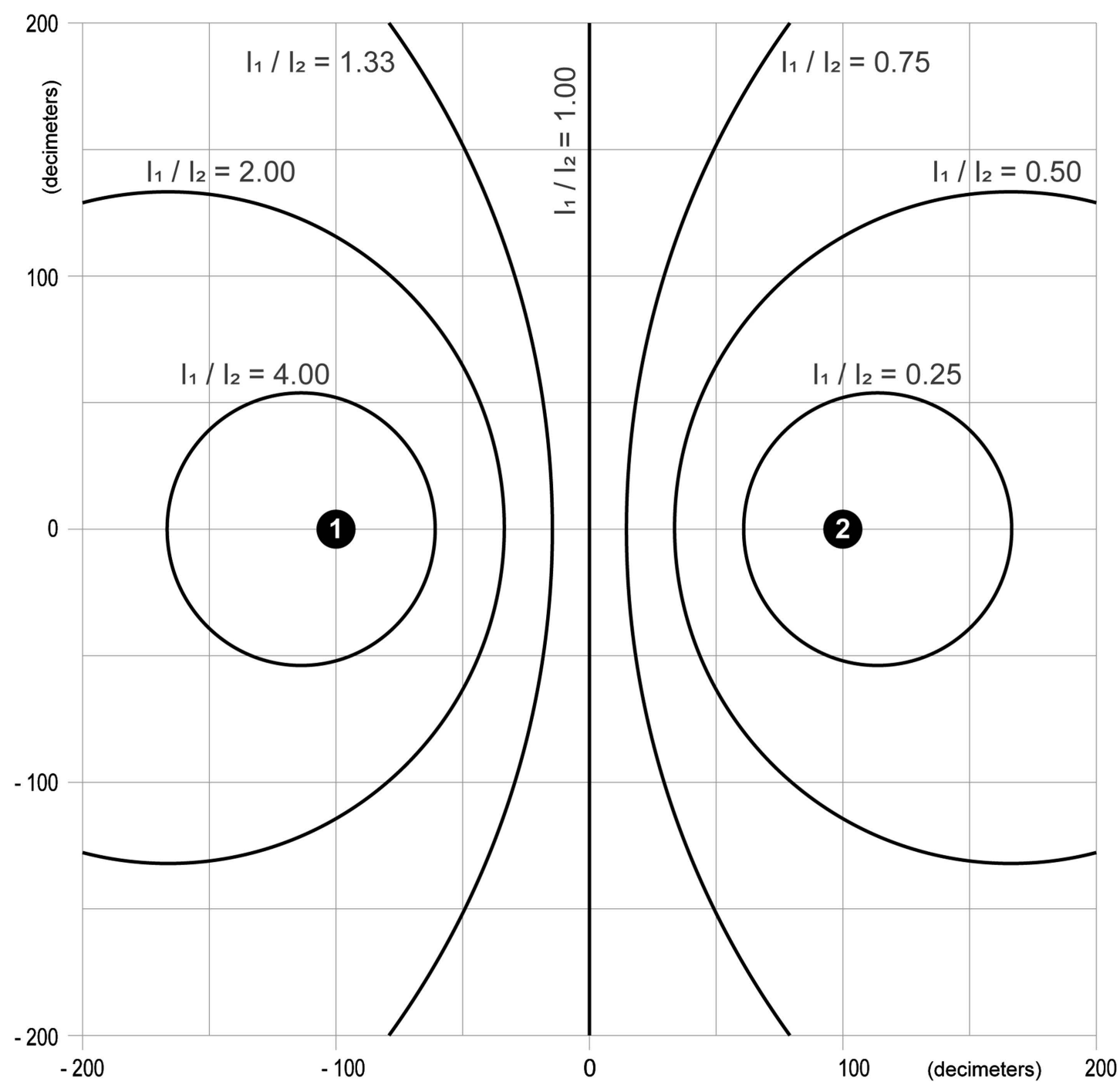

Figure 1. Set of examples for $I_{1} / I_{2}$ ratios. Number 1 and 2 in the figure mean the location of sensors number one and two.

$(-100.0)$ and the location of sensor $2=(100.0)$.

As shown above, whenever the ratio between the intensities measured is not 1 , the line is represented by an elliptic curve. Excluding some particular cases, the interception of two elliptic curves will be two points. So, a third independent curve is required to obtain one single interception point that represents the location of the source of the vibration:

$$
\begin{aligned}
& I_{1} r_{1}=I_{2} r_{2} \\
& I_{1} r_{1}=I_{3} r_{3} \\
& I_{1} r_{1}=I_{4} r_{4}
\end{aligned}
$$

A representation of the scenario described above is given by Figure 2. The interception point of three lines represents the location of the vibration source (Figure 2).

As shown above, a minimum of four sensor are needed to estimate the location 


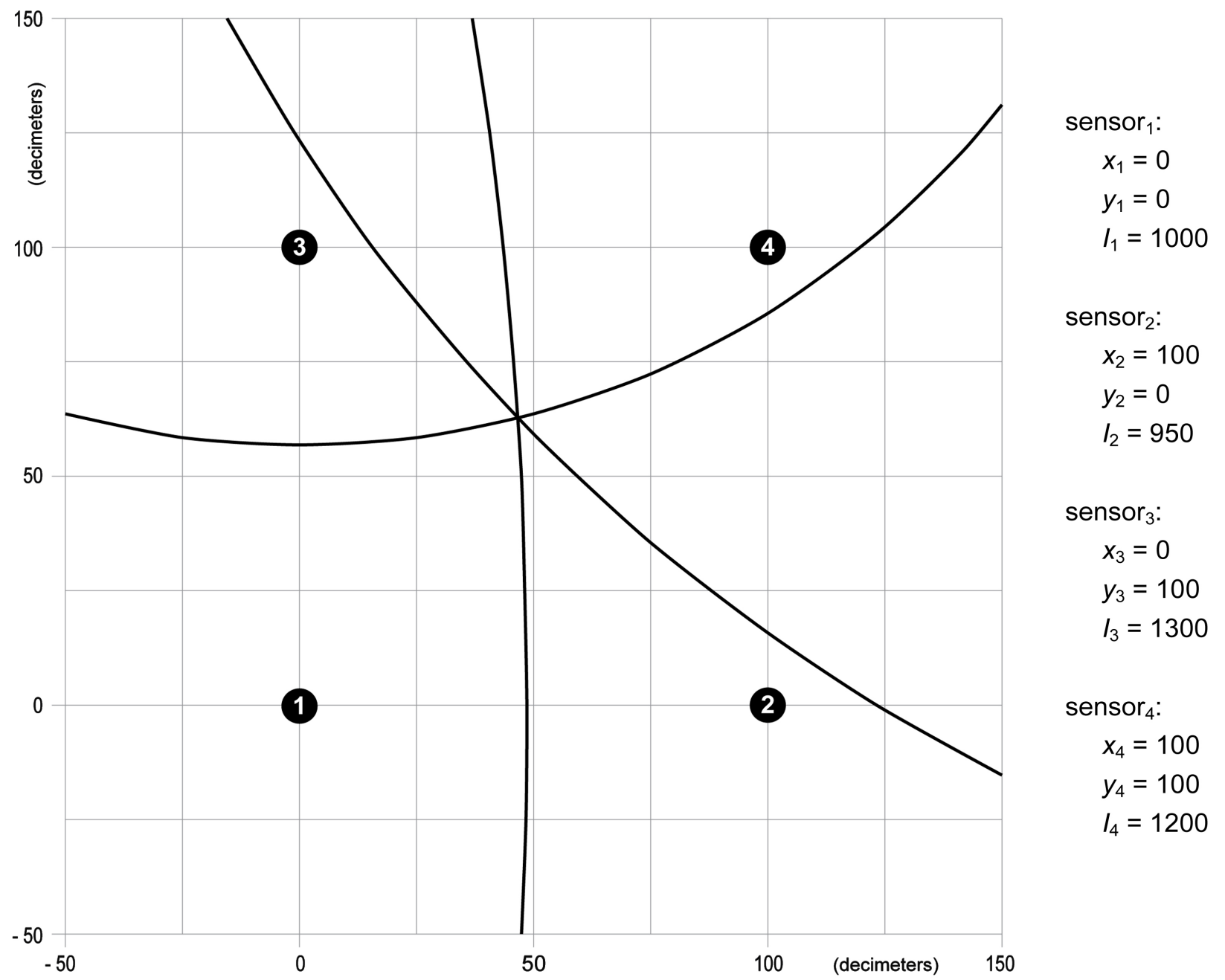

Figure 2. Example of an estimation of the vibration source location. Numbers 1, 2, 3, and 4 represents each sensor.

of the vibration source, and the vibration must be sensed in all of them. Whenever the lines interception does not represent an exact match (i.e. a single point), we will get a cloud of nearby points, obtained from the interception of each pair of lines. In this case, the average point represents the estimated location of the vibration source, and the distance to the furthest point will be the estimation error.

\subsection{Field Setup and Experimental Trials}

To test the new method proposed above, field trials were made which used four prototype vibration sensing units, developed specifically for this project. The diagram of each unit is shown in Figure 3, and specifications of the units are listed in Table 1.

The field setup consisted in turning on units 1, 2, 3 and 4, and placing them on the ground. Devices were connected to a portable PC, a Microsoft Surface Pro 3, connected to a Wireless LAN network named "iSense", and a SciLab version 5.5.2. was run (the custom application) do receive and show the signals 


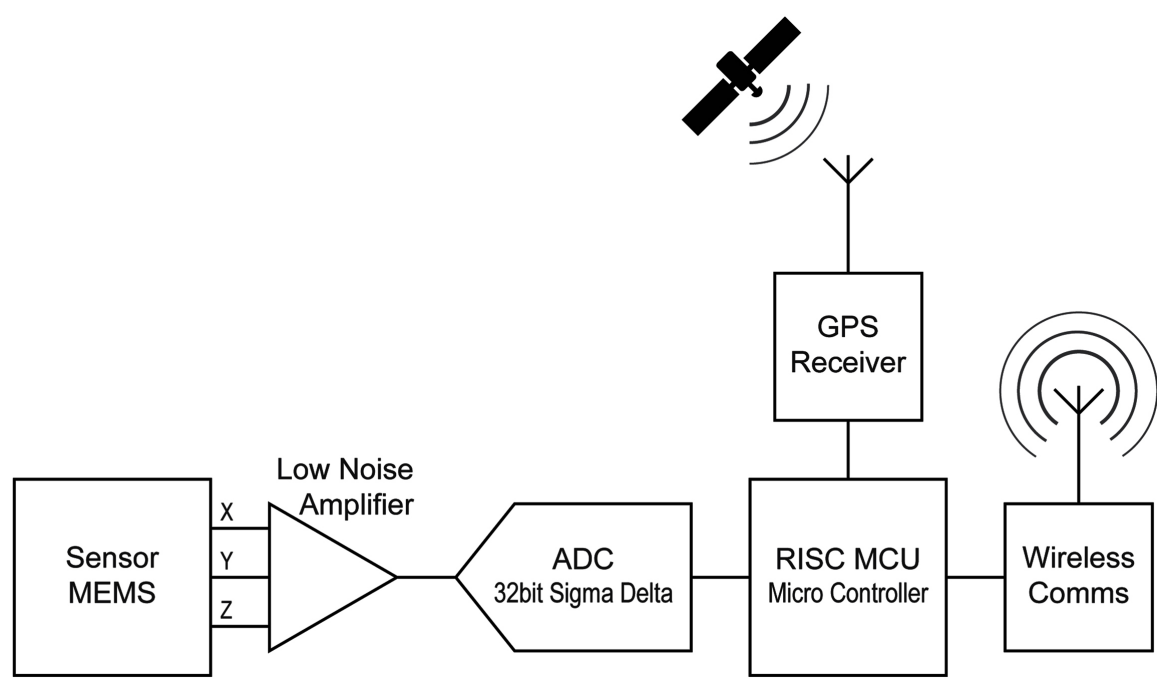

Figure 3. Sensor units' diagram: of the structure of the sensor, and of the detection system in each prototype.

Table 1. Technical specifications of prototype vibration sensing units.

\begin{tabular}{c}
\hline Issue \\
Power \\
Dimensions \\
Weight \\
Vibration sensing \\
Maximum acceleration sensing range \\
Lowest frequency sensitivity \\
Signal non-linearity \\
Signal noise density \\
Signal conversion \\
Sampling rate
\end{tabular}

Signal conversion integral nonlinearity (INL)

Low drift internal signal reference

Inter-axis interference (crosstalk)

Ultra-low signal distortion

Micro Controller Unit (MCU)

Wireless data communication

Antenas

GPS receiver

IP communications

$5 \mathrm{VDC}, 70 \mathrm{~mA}$

$90 \times 50 \times 17 \mathrm{~mm}$

60 grams

High-performance and low noise tri-axial MEMS accelerometer

$$
\begin{gathered}
\pm 2 \mathrm{~g} \\
0.001 \mathrm{~Hz} \\
\text { below } 0.1 \% \\
45 \mu \mathrm{g} / \mathrm{Hz}^{1 / 2}
\end{gathered}
$$

32 bits precision ( 1 bit represents $0.19 \mu \mathrm{g}$ ) with $>20$ noise free bits at 1000 sps

Programmable, 1000 sps as default $\pm 2.5 \mathrm{ppm}$ of full scale range (FSR)

$$
\begin{gathered}
2 \mathrm{ppm} /{ }^{\circ} \mathrm{C} \\
-120 \mathrm{~dB} \text { at } 1 \mathrm{kHz} \\
0.000022 \%
\end{gathered}
$$

Embedded; for local data processing

Wi-Fi $802.11 \mathrm{~b} / \mathrm{g} / \mathrm{n}$ with maximum transmission power of $+18 \mathrm{dBm}$

Embedded; $2.4 \mathrm{GHz}$

Embedded; 48 channels, signal sensitivity of -163 $\mathrm{dB} \cdot \mathrm{m}$, Accuracy lower than 2 meters for best scenario and time sync with an accuracy of $33 \mathrm{~ns}$ (good conditions)

With a data stream rate of $256 \mathrm{ksps}$ for $1000 \mathrm{sps}$ signal sampling rate 
from the units.

\subsection{Field Trials}

We performed field trials to collect data that allowed us to analyse the following parameters: 1) Maximum sensing distance determination of the prototypes; 2) Location source vibration (math calculation); and 3) Identification of distinct signal patterns of ground vibrations.

The trials were conducted in the winter season (air temperature ranged from $9^{\circ} \mathrm{C}-12^{\circ} \mathrm{C}$, and soil humidity was approximately $90 \%$ ) in 2016 in a pine forest with stabilised sand soil from dunes (modern sedimentary deposits) at the following coordinates $40^{\circ} 34^{\prime} 41.6^{\prime \prime} \mathrm{N} 8^{\circ} 43^{\prime} 54.2^{\prime \prime} \mathrm{W}$ (place 1), and in 2017 , at the following coordinates $40^{\circ} 33^{\prime} 55.73^{\prime \prime N} ; 8^{\circ} 29^{\prime} 42.86^{\prime \prime W}$ (place 2), Aveiro, Portugal. This second location corresponds to a soccer game field with a homogeneous floor of pliocene-pleistocene sands and the Triassic Eirol sandstone [14] as base rock of this field. To ensure the accurate relative locations for all sensors and simulated source-vibration, we used a measure tape (with 20 meters as maximum length).

In each field trial, and with the propose to create a standard ground vibration signal, we repeated the dropping of the $8 \mathrm{~kg}$ mass, from a height of about $1 \mathrm{me}-$ ter from the soil, to simulate a vibration source. For each spot marked to drop of the mass, we repeated three times the procedure. This was done in experiments with the four prototypes in line, or in a square distribution setup on the floor. Also, the ground-signals generated by an $80 \mathrm{~kg}$ running man were collected by the prototypes. A third kind of signals were generated by the reproduction of audio record [15] by a FIIO device, X1 model coupled to a Subwofer Logitec Z-4i 2.1 speaker attached to the ground, to simulate an elephant sound propagation over the ground.

\subsection{Maximum Sensing Distance}

To test the maximum sensing distance, we placed one sensor on the floor and simulated a sequence of vibrations at a known distance from the sensor (Figure 4) and, at each point three impacts were repeated.

\subsection{Location of the Source-Vibration}

To estimate the location of the signal source we need to know the relative distances between the units and to receive clear signal in the four units. The field trial described in Figure 5 was repeated in three sequences. We applied the model described to estimate the location of the vibration source, as well as the maximum error for that estimation. For the location of the vibration source trial, the four sensors were placed as shown in the diagram of Figure 5.

\subsection{Identification of Distinct Signal Patterns of Ground Vibrations: Spectral Signature}

To assess the spectral diversity of the signals according with the type of the signal 


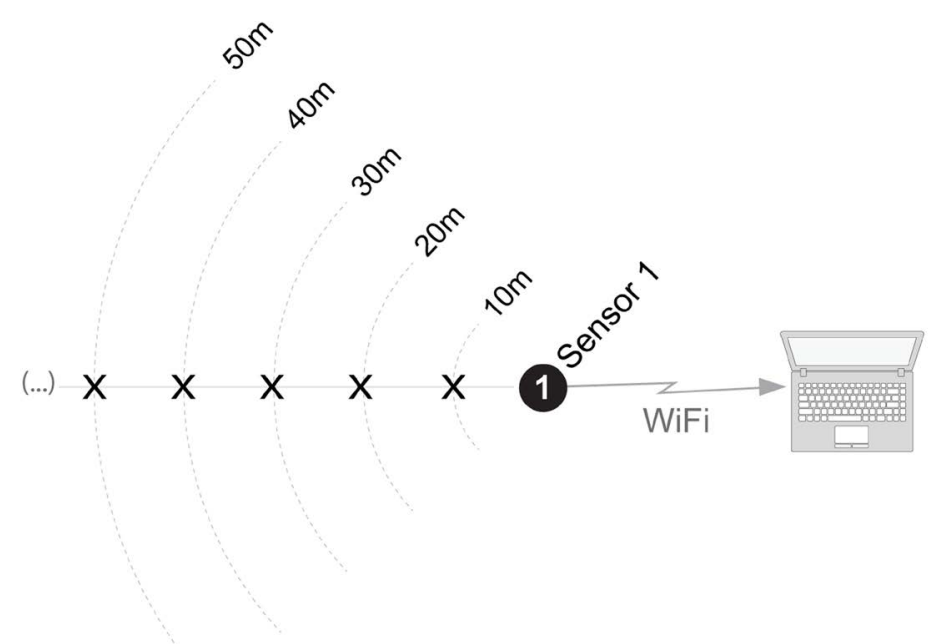

Figure 4. Diagram showing the field tests setup.

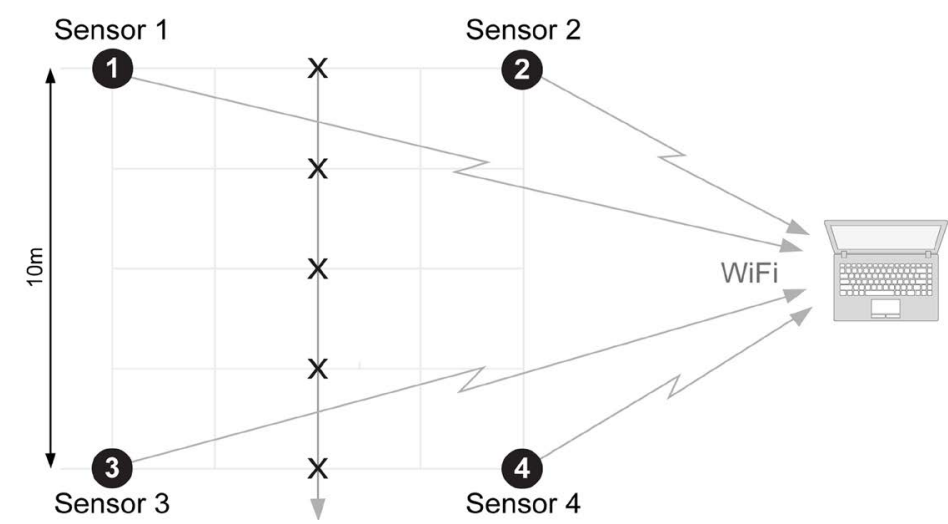

Figure 5. Field tests setup for the location of the vibration source. The $\mathrm{X}$ in the figure, represents the spots (points) where the signal was generated (place 2).

source, we used the same field, and simulated a vibration episode using different sources, such us, the drop of an 8 kilogram weight, a man running, and the reproduction of African savannah elephants recorded sound [15] using a vibration speaker attached to the ground. The spectral signatures data provides, helps in assessing the potential for movement detection and identification of a source (animal, human, or other).

\section{Results}

\subsection{Maximum Sensing Distance}

The distance sensing performance of the units for several different signal sources, and the unit setup is shown in Figure 6. For the $8 \mathrm{~kg}$ mass drop scenario, the maximum sensing distance can go from 25 to approximately 52 meters (Figure 6).

The graph shows that a man running causes lower soil vibrations, when compared with the $8 \mathrm{~kg}$ mass weight. The maximum sensing distance is 


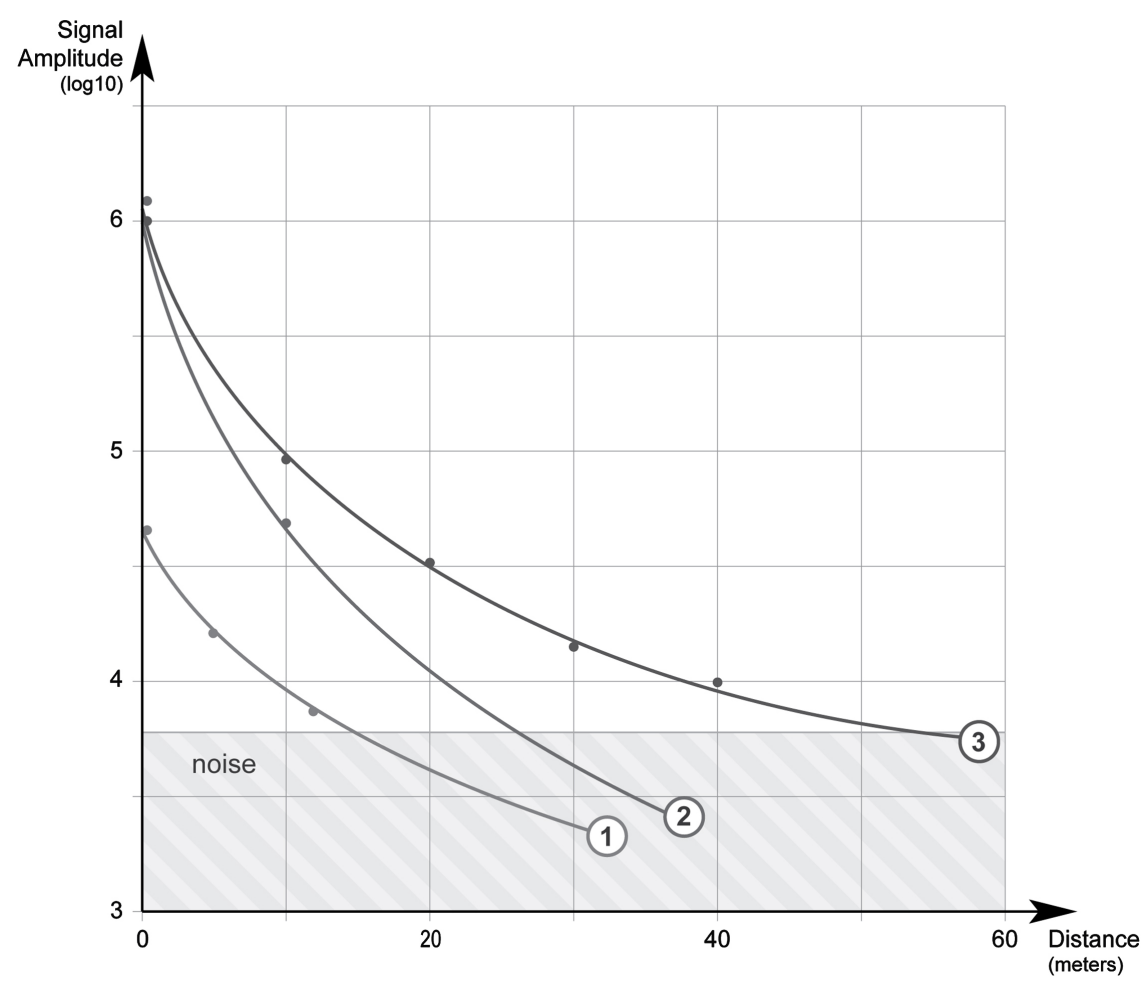

Figure 6. Signal amplitude variation with distance to the vibration source: runner (curve 1, place 1), $8 \mathrm{~kg}$ mass drop (curve 2, place 2; curve 3, place 1). The gray area in the figure represents the background noise signal.

approximately 15 meters for this setup.

The experimental data is consistent with the theoretical model of the mechanical wave's intensity (the equation number 1 ).

$$
I_{n}=\frac{I_{\text {source }}}{\pi r^{2}}
$$

\subsection{Location of the Source-Vibration}

The following diagrams of the Figure 7 show the accuracy between the location of the impact point of the vibration source and the obtained by the sensors.

Considering the estimated errors obtained above for each point, the global average error is \pm 0.232 meters. Having in consideration the dimension of this array of sensors, with an interval of 10 meters between sensors, the average error is $4.64 \%$ of the size of the sensors array.

\subsection{Identifying Distinct Signal Patterns}

Using the signals collected from the field trial, we obtained the spectrograms shown in Figures 8-10.

As shown in the spectrograms (Figures 8-10), distinctive and unique patterns are gotten for the different events. The data obtained from the field trials also showed the repeatability of this pattern so we can establish a relation between the pattern and the event. For the purpose of this solution, a library of signatures 
will be required to allow the system to recognize events along the virtual fence. This library can be developed using a machine learning approach, that is, continuous expansion of the library by cross-checking unknown events.

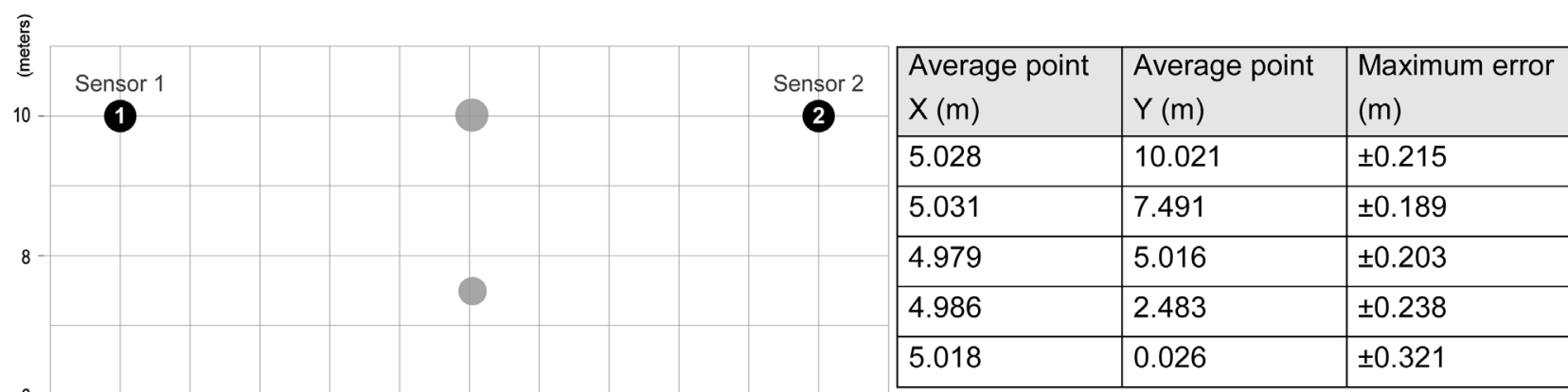

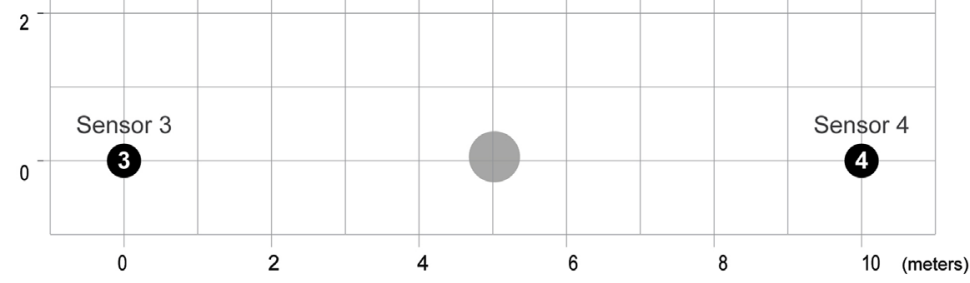

(a)

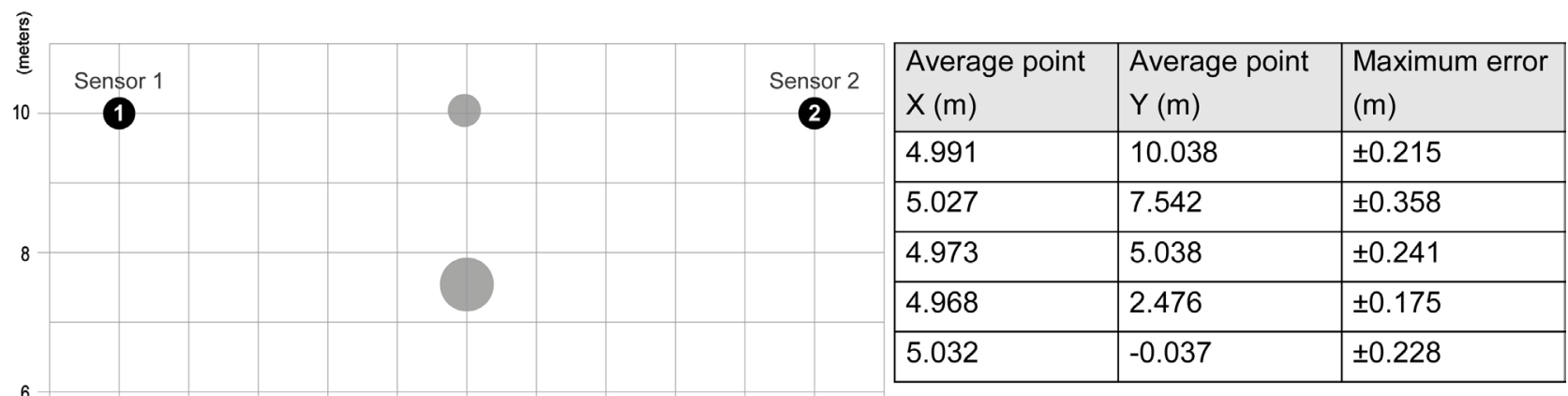

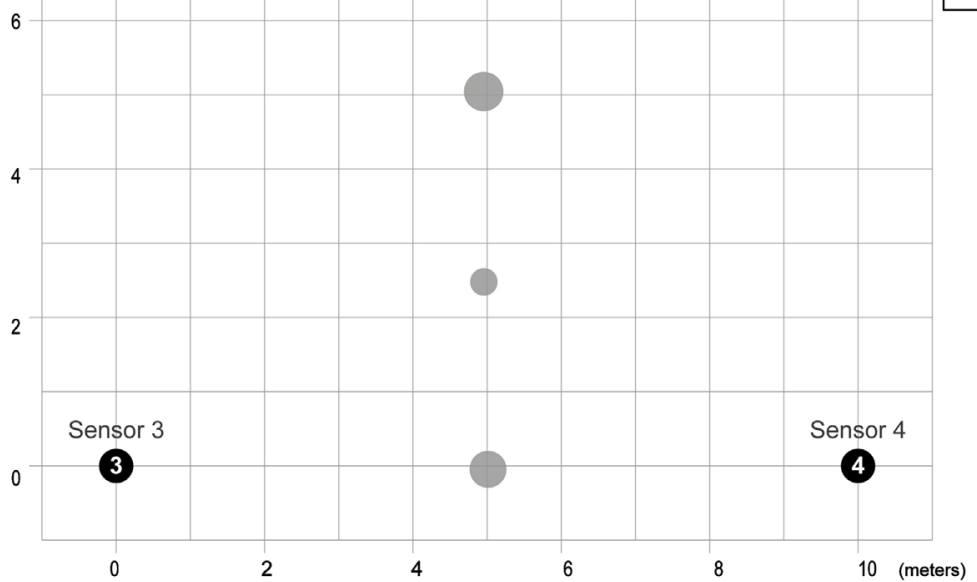

(b) 


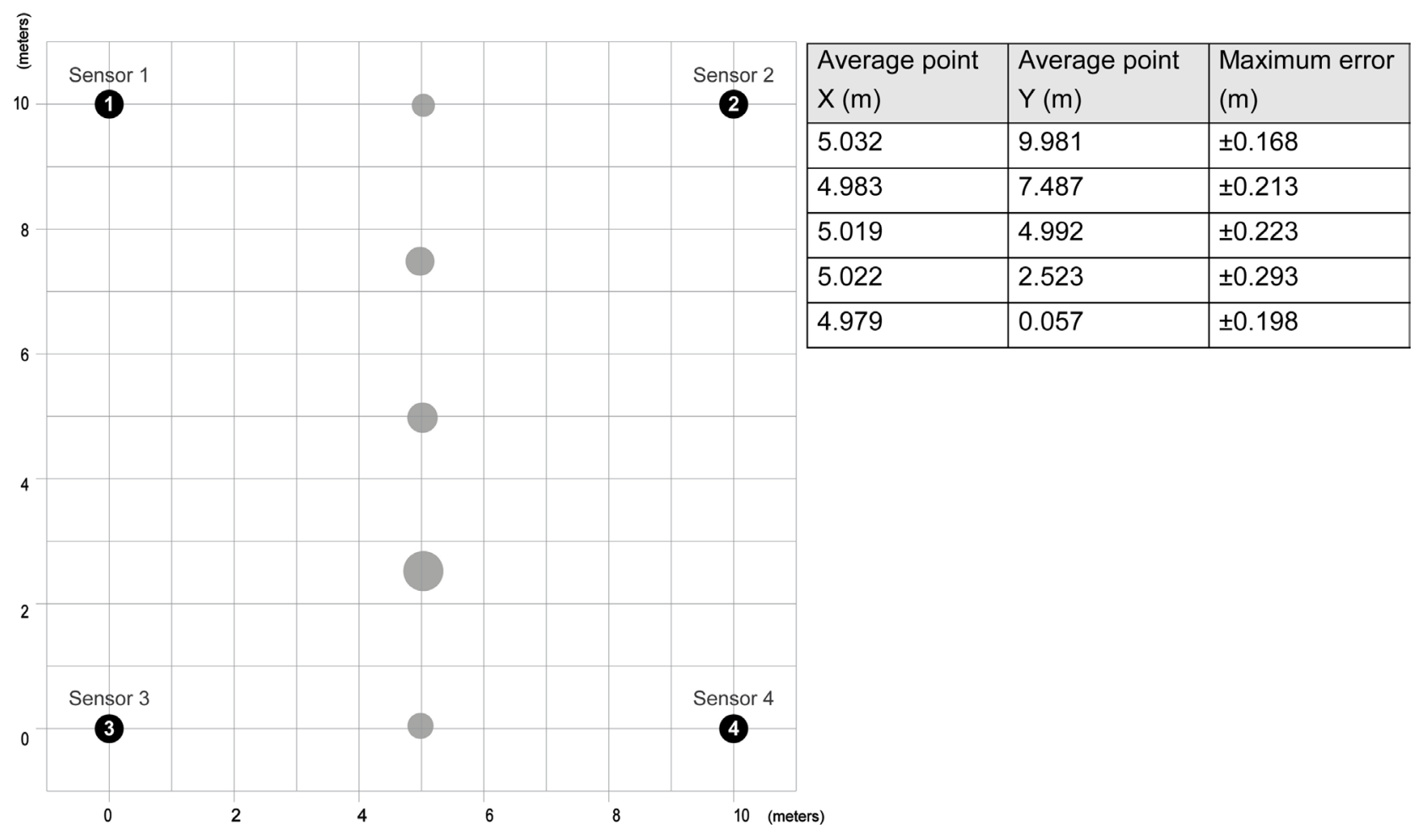

(c)

Figure 7. (a) Results obtained from the first sequence of the field trial; (b) Results obtained from the second sequence of the field trial; (c) Results obtained from the third sequence of the field trial (place 2).

\section{Discussion}

MEMS-based digital sensors have their advantages, since these sensors offer new capabilities compared with conventional arrays of geophones, because they "provide better vector fidelity thanks to its accurate calibration (amplitude and orthogonality), broadband linear response (from DC to $800 \mathrm{~Hz}$ ) and low distortion $(<-90 \mathrm{~dB})$ "; also integration of the sensor with the station electronics allows size/weight reduction provide complete digital transmission, from the sensor to the central unit, which is less sensitive to electromagnetic pick-up, cross-talk, and leakage offers the potential to reduce costs while improving data quality [13]. Having in mind the need to address human-wildlife conflict (HWC), and to overcome the limitations and issues of the traditional solutions, this work focused on developing the "loxophone" device.

HWC is a critical aspect of any wildlife conservation initiative. From the human perspective and as referred in Woodroffe et al. (2014) [16], this conflict often involves the damage of goods (valuable livestock, crops, or infrastructure), carry of diseases and risk for human lives. In other hand, human pressure over wildlife causes degradation of wildlife habitat with significant risk for reduction or local extinction of their wildlife species.

Pitman et al. (2017) [17] shows that the mitigation mechanism to prevent HWC, not only promotes (and is highly effective in) the protection of wildlife species, but can ramp-up local economies with great benefits for local populations. 


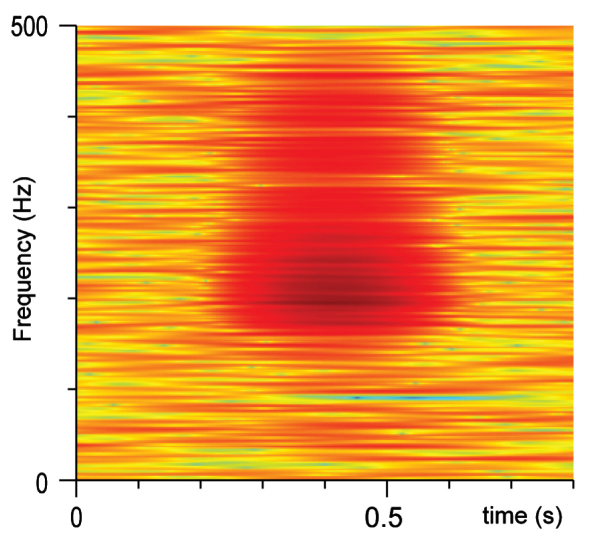

(a)

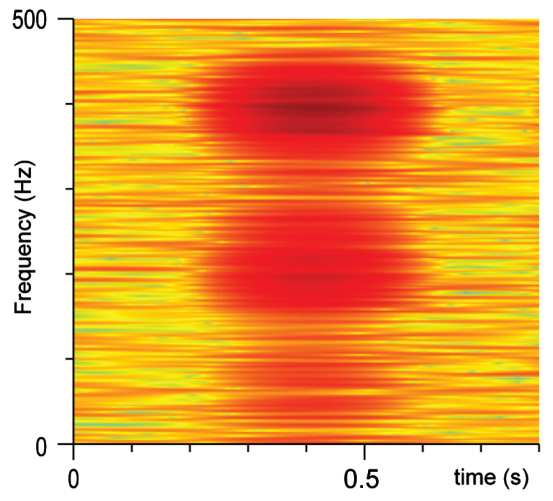

(b)

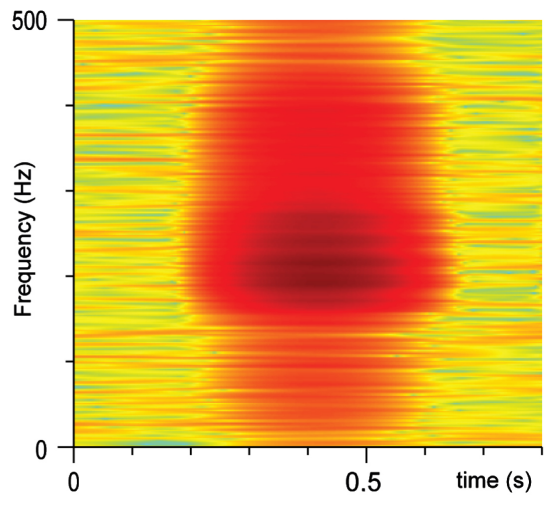

(c)

Figure 8. Spectrograms of an $8 \mathrm{~kg}$ weight drop off (in free fall). (a) X axis; (b) Y axis; (c) $\mathrm{Z}$ axis (place 1$)$.

To mitigate this conflict, fencing has been a widely used approach to define the borders of protected areas. The physical solid barrier created by the fences, has proven to be an effective way to enforce the separation between humans and wildlife. However, and as discussed by Woodroffe et al. (2014) [16], fencing comes with some drawbacks, namely, habitat fragmentation with direct impact on the local ecosystem balance. Also fencing costs are high [18] [19], limiting the feasibility of fencing of large or very large protection areas.

The "loxophone" solution provides: an affordable cost, since it uses nowadays technology, and avoids expensive geophone sensors; wireless mesh network, avoiding the need of long cables and time consuming installations; high sensitivity, since the MEMS sensor is a high resolution Analog to Digital Converter to capture very low vibrations; 3 axis analysis to improve sensing capability for all waves independently of the polarity of the wave when crossing the sensor, contrasting with mono axial sensors; GPS data to identify the location of the sensor and provide time synchronization, and to support triangulation calculus to determine location of the source-vibration; a viable solution to implement medium to large size geofences, since traditional solution are expensive and complex for such scale. As potentialities for this technology the research team became aware 


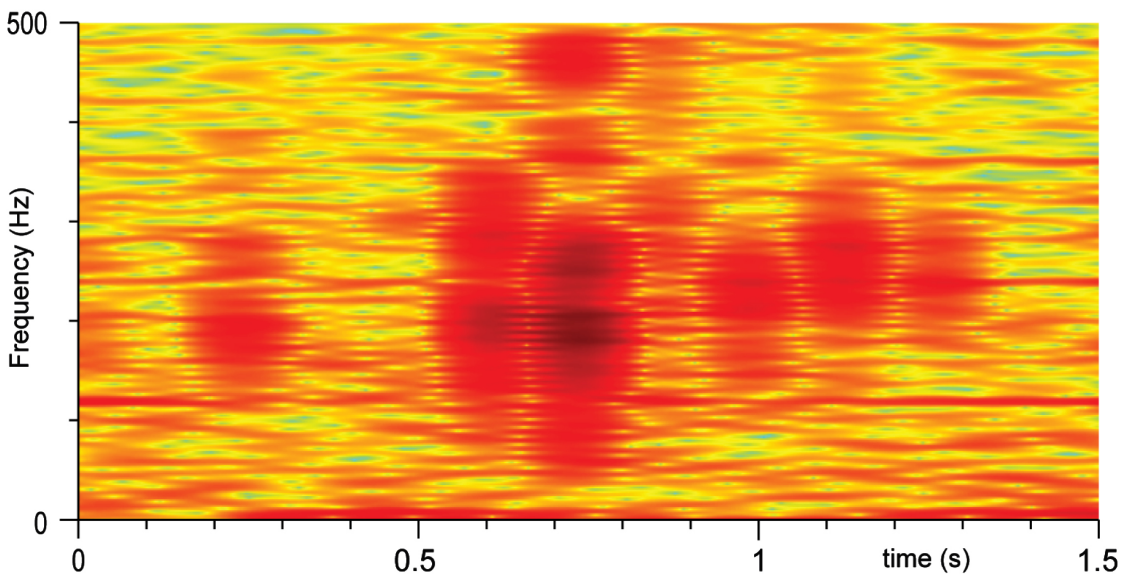

(a)

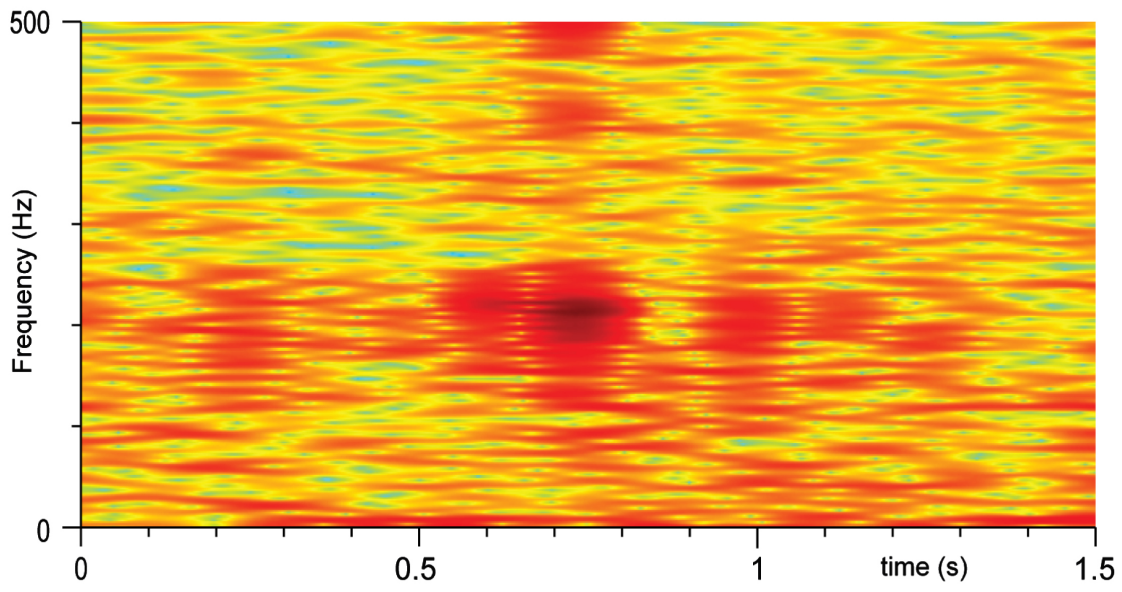

(b)

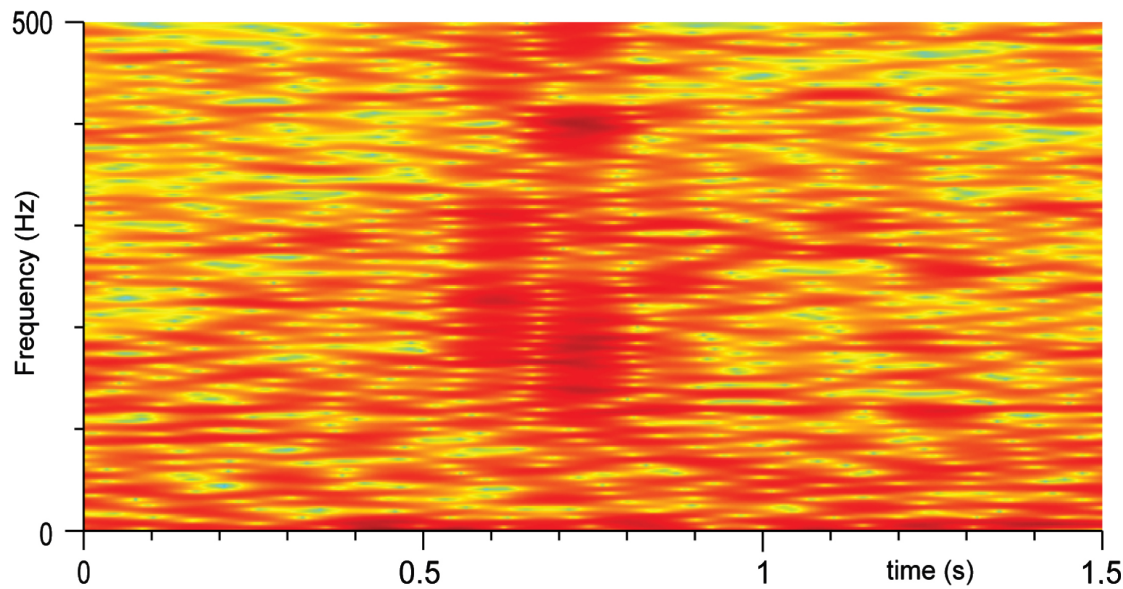

(c)

Figure 9. Spectrograms of a man running. (a) $\mathrm{X}$ axis; (b) $\mathrm{Y}$ axis; (c) $\mathrm{Z}$ axis (place 1).

that vibration sources produce spectral signatures that enable the identification of the vibration cause; e.g. if an animal produces a specific signature, it is possible not only to know "where" it is, but also "what" is crossing the virtual fence. As shown on results, we can obtain distinctive spectral signatures that can be 


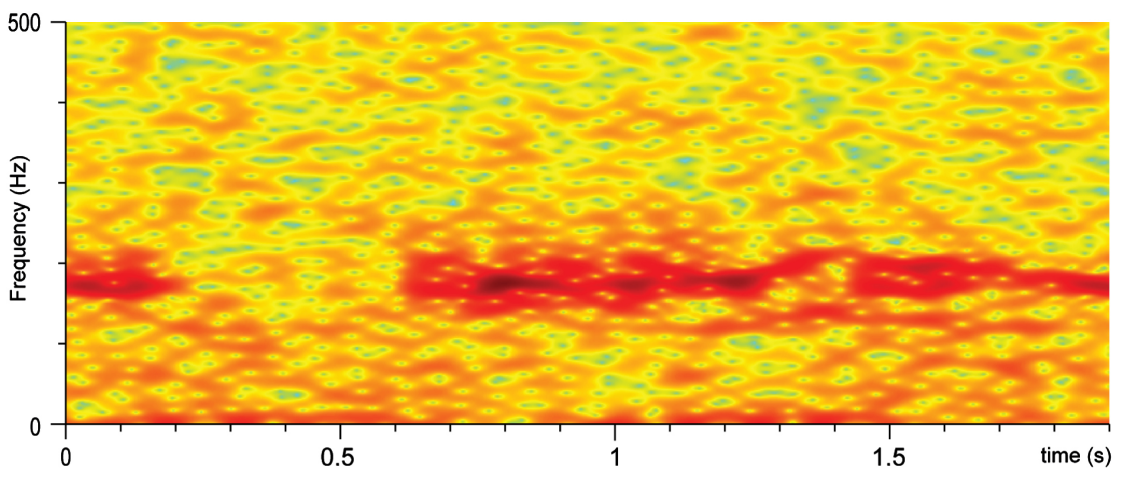

(a)

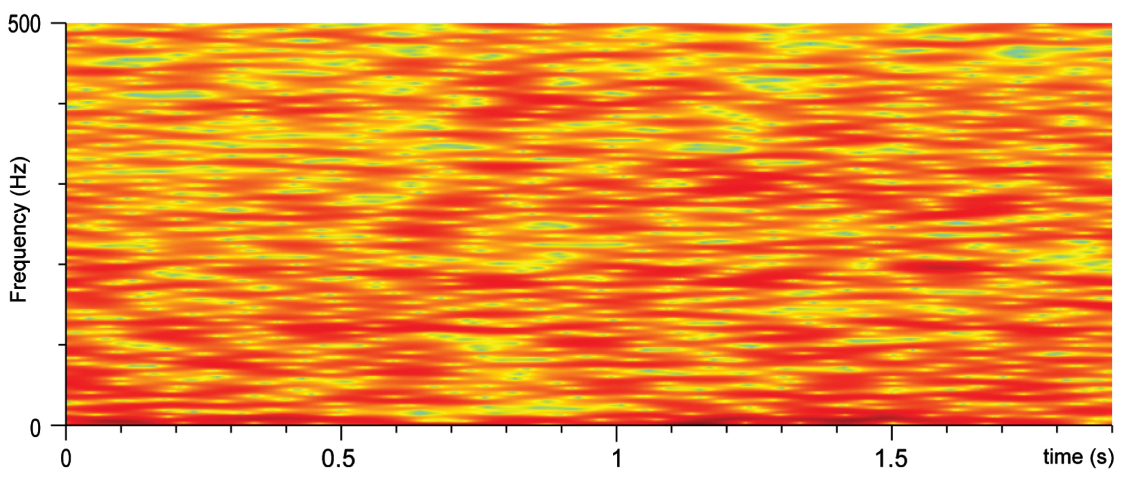

(b)

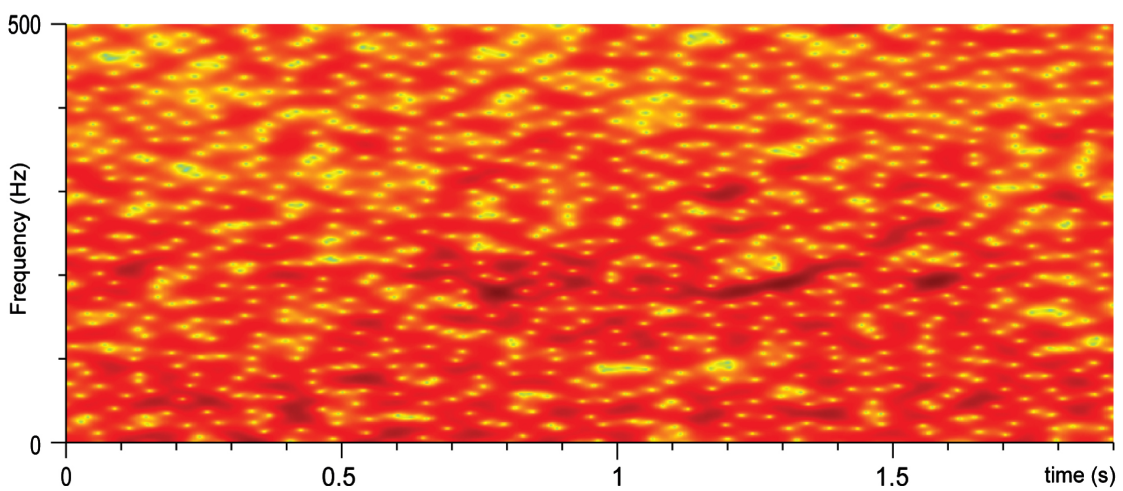

(c)

Figure 10. Spectrograms of elephant's record sounds to trigger a vibration source. (a) $\mathrm{X}$ axis; (b) Y axis; (c) $\mathrm{Z}$ axis (place 1).

related with different events and vibration sources. Günther et al. (2004) [5] and Mehmood et al. (2012) [20] show this same conclusion about unique spectral signature using traditional single-axis geophone sensors. This approach enables the free-ranging of wildlife and humans because this virtual fence is not intrusive by default. This means that it is required an action mechanism associated to react to the real-time alerts triggered by this solution. As example of action mechanisms, we can have field teams, local visual and/or sound alert signals or unmanned aerial vehicle, like autonomous Quadcopters, to take-off and fly to the incident location and take some action. 
With this solution, the location and identification not only of the large size wildlife, but of pouching activities is possible.

\section{Conclusions}

The field trials conducted with these prototype sensors shown that we can locate the vibration source with an error below 5\%. Considering the objective of this solution, we believe that this provides a good level of accuracy to track events along the virtual fence.

We found from the results above that the location accuracy and system sensitivity is dependent on the distance between sensors. So, the shorter is the distance between sensors, higher will be the level of sensitivity and accuracy of the virtual fence, but more sensors per kilometre will be required.

Considering the sensing distance obtained from the trials, we believe that an interval between sensors of about 25 meters will provide enough sensitivity and accuracy to detect and track large size wild animals, such us elephants. For this solution scenario, we forecast that the sensors network mesh would cost less than $25 \%$ of the traditional fencing costs per kilometre. This forecast, based on components and industrial process costs simulation, allows us to offer an effective virtual fence at a cost that would enable medium to large fencing.

\section{Author Contributions Statement}

R.A., S.C. and M.J.P. collaborated from the first stage of the investigation, making contributions to test the equipment, data acquisition, data analysis, and writing the manuscript. S.C. developed the hardware device, the SciLab instructions set and the mathematical model.

\section{Statement}

This project was supported by the authors whom disclaim any conflict of interests.

\section{Competing Financial Interests}

The intellectual property of the prototypes belongs to S.C. and he owns the rights to use this solution in commercial applications.

\section{References}

[1] Arosio, D., Longoni, L., Papini, M., Scaioni, M., Lanzi, L. and Alba, M. (2009) Towards Rockfall Forecasting through Observing Deformations and Listening to Microseismic Emissions. Natural Hazards and Earth System Sciences, 9, 119-1131. http://www.nat-hazards-earth-syst-sci.net/9/1119/2009/ https://doi.org/10.5194/nhess-9-1119-2009

[2] Collins, D.S., Toya, Y., Hosseini, Z. and Trifu, C.-I. (2014) Real Time detection of Rock Fall Events Using a Microseismic Railway Monitoring System. Geohazards6, Kingston.

[3] Senfaute, G., Duperret, A. and Lawrence, J.A. (2009) Micro-Seismic Precursory 
Cracks Prior to Rock-Fall on Coastal Chalk Cliffs: A Case Study at Mesnil-Val, Normandie, NW France. Natural Hazards and Earth System Sciences, 9, 1625-1641. https://doi.org/10.5194/nhess-9-1625-2009

[4] Zimmer, V.L. and Sitar, N. (2015) Detection and Location of Rocks Falls Using Seismic and Infrasound Sensors. Engineering Geology, 193, 49-60.

https://doi.org/10.1016/j.enggeo.2015.04.007

[5] Günther, R.H., O’Connell-Rodwell, C.E. and Klemperer, S.L. (2004) Seismic Waves from Elephant Vocalizations: A Possible Communication Mode? Geophysical Research Letters, 31, L11602. https://doi.org/10.1029/2004GL019671

[6] O’Connell-Rodwell, C.E. (2007) Keeping an "Ear” to the Ground: Seismic Communication in Elephants. Physiology, 22, 287-294.

https://doi.org/10.1152/physiol.00008.2007

[7] Prince, J.N. and Sugumar, S.J. (2014) Surveillance and Tracking of Elephants Using Vocal Spectral Information. IJRET, 3, 664-671.

https://doi.org/10.15623/ijret.2014.0319118

[8] Zeppelzauer, M. and Stoeger, A.S. (2015) Establishing the Fundamentals for an Elephant Early Warning and Monitoring System. BMC Research Notes, 8, 409. https://doi.org/10.1186/s13104-015-1370-y

[9] Liang, T. and Lin, Y. (2013) A Fiber-Optic Sensor for the Ground Vibration Detection. Optics Communications, 306, 190-197. https://doi.org/10.1016/j.optcom.2013.05.057

[10] Jewett, J. and Serway, R. (2007) Physics for Scientists and Engineers. 7th Edition, Brooks/Cole, Pacific Grove.

[11] Kearey, P., Brooks, M. and Hill, I. (2002) An Introduction to Geophysical Exploration. 3rd Edition, Blackwell Science, Ltd., Oxford.

[12] Kamei, R., Nakata, N. and Lumley, D. (2015) Introduction to Microseismic Source Mechanisms. The Leading Edge, 34, 876-880. https://doi.org/10.1190/tle34080876.1

[13] Lainé, J. and Mougenot, D. (2014) A High-Sensitivity MEMS-Based Accelerometer. The Leading Edge, 33, 1234-1242. https://doi.org/10.1190/tle33111234.1

[14] Teixeira, C. and Zbyszewski, G. (1976) Carta Geológica de Portugal na escala 1/50000. Notícia explicativa da folha 16-A (Aveiro), Serviços Geológicos de Portugal, Lisboa.

[15] Stoeger, A.A. and Manger, P. (2014) Vocal Learning in Elephants: Neural Bases and Adaptive Context. Current Opinion in Neurobiology, 28, 101-107. https://doi.org/10.1016/j.conb.2014.07.001

[16] Woodroffe, R., Hedges, S. and Durant, S.M. (2014) To Fence or Not to Fence. Science, 344, 46-48. https://doi.org/10.1126/science.1246251

[17] Pitman, R.T., Fattebert, J., Williams, S.T., Williams, K.S., Hill, R.A., Hunter, L.B.T., Slotow, R. and Balme, G.A. (2017) The Conservation Costs of Game Ranging. Conservation Letters, 10, 403-413. https://doi.org/10.1111/conl.12276

[18] Evans, L.A. and Adams, W.M. (2016) Fencing Elephants: The Hidden Politics of Wildlife Fencing in Laikipia, Kenya. Land Use Policy, 51, 215-228. https://doi.org/10.1016/j.landusepol.2015.11.008

[19] Huijser, M.P., Duffield, J.W., Clevenger, A.P., Ament, R.J. and McGowen, P.T. (2009) Cost-Benefit Analyses of Mitigation Measures Aimed at Reducing Collisions with Large Ungulates in the United States and Canada; A Decision Support Tool. Ecology and Society, 14, 15. https://www.ecologyandsociety.org/vol14/iss2/art15/ 
https://doi.org/10.5751/ES-03000-140215

[20] Mehmood, A., Damarla, T. and Sabatier, J. (2012) Separation of Human and Animal Seismic Signatures using Non-Negative Matrix Factorization. Pattern Recognition Letters, 33, 2085-2093. https://doi.org/10.1016/j.patrec.2012.06.015 